PROCEEDINGS OF THE

AMERICAN MATHEMATICAL SOCIETY

Volume 124, Number 3, March 1996

\title{
ON PREPONDERANT DIFFERENTIABILITY OF TYPICAL CONTINUOUS FUNCTIONS
}

\author{
L. ZAJÍČEK
}

(Communicated by C. D. Sogge)

\begin{abstract}
In the literature, several definitions of a preponderant derivative exist. An old result of Jarník implies that a typical continuous function on $[0,1]$ has a (strong) preponderant derivative at no point. We show that a typical continuous function on $[0,1]$ has an infinite (weak) preponderant derivative at each point from a $c$-dense subset of $(0,1)$.
\end{abstract}

\section{INTRODUCTION}

Let $C$ denote the set of continuous real functions defined on $[0,1]$ furnished with the metric of uniform convergence. As usual, when we say a typical $f \in C$ has a certain property, we mean that the set of $f \in C$ with this property is residual in $C$. References concerning differentiability properties of typical continuous functions can be found in [2] and [11]. The first results of this type ([1], [7]) imply that a typical $f \in C$ has a derivative at no point. A result of Jarník [5] (cf. [11], Theorem J, (iii)) immediately implies that a typical $f \in C$ has a preponderant derivative (finite or infinite) at no point of $(0,1)$, if we use a strong definition of a preponderant derivative (cf. Definition 1 below) which was used e.g. by Bruckner [3]. The same result holds true if we consider a slightly weaker definition of a preponderant derivative used in [8].

The main result of the present article says that the situation changes if we use another weaker notion of a preponderant derivative (cf. Definition 2 below), which is still stronger than the notion of a preponderant derivative used by Denjoy [4]. Namely we prove that a typical function $f \in C$ has this weaker preponderant derivative $f_{p r}^{\prime}(x)=\infty$ at each point $x$ from a $c$-dense subset of $(0,1)$. Note that the infiniteness of the preponderant derivative is essential, since a theorem of Jarník [5] (cf. [11], Theorem J, (ii)) immediately implies that a typical $f \in C$ has at no point a finite preponderant derivative (any existing definition of a preponderant derivative can be used here). Further note that a slightly weaker version of the main result of the present article is stated without a proof in [11] (Theorem 4, (ii)). The main tools for the proof of our result is the Banach-Mazur game and an unusual monotonicity theorem of [6].

Received by the editors March 15, 1994 and, in revised form, August 23, 1994.

1991 Mathematics Subject Classification. Primary 26A24.

Key words and phrases. Preponderant derivative, typical continuous function, Banach-Mazur game.

Supported by Research Grants GAUK 363 and GAČR 0474. 


\section{Preliminaries}

If $a>b$, then the symbol $(a, b)$ will denote the open interval $(b, a)$. The symbol $\mu$ will denote the Lebesgue measure on $\mathbb{R}$. The open ball in a metric space $P$ with the center $c \in P$ and the radius $r>0$ is denoted by $B(c, r)$.

The definition of the strong preponderant derivative used in [3] and mentioned in the Introduction is the following.

Definition 1. We say that $A$ is a strong preponderant derivative of a function $f$ at a point $a \in \mathbb{R}$ if there exists a measurable set $E \subset \mathbb{R}$ such that

$$
\underline{\lim }_{h \rightarrow 0+} \frac{\mu(E \cap(a, a+h))}{h}>\frac{1}{2}, \quad \underline{\lim }_{h \rightarrow 0+} \frac{\mu(E \cap(a-h, a))}{h}>\frac{1}{2}
$$

and

$$
\lim _{x \rightarrow a, x \in E} \frac{f(x)-f(a)}{x-a}=A .
$$

We shall work with the following weaker definition.

Definition 2. We say that a function $f$ has at a point $a$ the preponderant derivative $f_{p r}^{\prime}(a)=A$ if there exists a measurable set $E \subset \mathbb{R}$ such that

$$
\lim _{x \rightarrow a, x \in E} \frac{f(x)-f(a)}{x-a}=A
$$

and $\frac{\mu(E \cap I)}{\mu(I)}>\frac{1}{2}$ for every interval $I$ containing $a$.

One of the main tools of the present article is the Banach-Mazur game. It is the following infinite game between two players.

Let $P$ be a metric space and let $Q \subset P$ be given. In the first step the first player chooses an open ball $B\left(g_{1}, \delta_{1}\right)$. In the second step the second player chooses an open ball $B\left(f_{1}, \varepsilon_{1}\right) \subset B\left(g_{1}, \delta_{1}\right)$, in the third step the first player chooses an open ball $B\left(g_{2}, \delta_{2}\right) \subset B\left(f_{1}, \varepsilon_{1}\right)$, and so on. If

$$
\bigcap_{i=1}^{\infty} B\left(f_{i}, \varepsilon_{i}\right) \subset Q,
$$

then the second player wins. In the opposite case the first player wins.

We shall need the following theorem essentially due to Banach.

Theorem BM. The second player has the winning strategy in the Banach-Mazur game if and only if $Q$ is a residual subset of $P$.

A proof of this theorem can be found in $[10]$ in the case $P=(0,1)$; the proof in the general situation (cf. [9]) is essentially the same.

The second essential tool is the following basic lemma which is an easy consequence of the main result of [6]. 
Lemma 1. Let $\varphi$ be a continuous function on $[a, b]$, let $S$ be a countable set and let $\frac{\varphi(b)-\varphi(a)}{b-a}>K$. Then there exists $x \in(a, b) \backslash S$ for which

$$
\begin{gathered}
\mu\left\{z \in(x, y): \frac{\varphi(z)-\varphi(x)}{z-x}>K\right\}>\frac{1}{2}(y-x) \text { for all } y \in(x, b] \text { and } \\
\mu\left\{z \in(y, x): \frac{\varphi(z)-\varphi(x)}{z-x}>K\right\}>\frac{1}{2}(x-y) \text { for all } y \in[a, x) .
\end{gathered}
$$

Proof. Choose $\varepsilon>0$ such that $\varphi(b)-\varphi(a)>(K+\varepsilon)(b-a)$ and consider the function $f(x):=(K+\varepsilon) x-\varphi(x)$. Since $f(b)<f(a)$ and the conditions (B), (C), (D) of Theorem 1 from [6] are clearly satisfied for $\alpha=1 / 2$, we obtain that (A) does not hold. Consequently there exists $x \in(a, b) \backslash S$ such that

(3) $\mu\{z \in(x, y):(K+\varepsilon) z-\varphi(z)>(K+\varepsilon) x-\varphi(x)\}<\frac{1}{2}(y-x)$ for all $y \in(x, b]$ and

(4) $\mu\{z \in(y, x):(K+\varepsilon) z-\varphi(z)>(K+\varepsilon) x-\varphi(x)\} \geq \frac{1}{2}(x-y)$ for all $y \in[a, x)$.

It is easy to see that (3) immediately implies (1). Since $f$ is continuous, it is easy to prove that (4) implies (2).

We shall also need the following two easy lemmas.

Lemma 2. Let $f$ be a measurable real function, let $x \in \mathbb{R}$ and let $a_{n} \nearrow x$ and $b_{n} \searrow x$ be strictly monotone sequences such that, for all natural numbers $n$,

$$
\mu\left\{z \in(y, x): \frac{f(z)-f(x)}{z-x}>n\right\}>\frac{|x-y|}{2}
$$

whenever $y \in\left[a_{n}, a_{n+1}\right]$ or $y \in\left[b_{n+1}, b_{n}\right]$. Then $f_{p r}^{\prime}(x)=\infty$.

Proof. Fix a natural $n$. The function

$$
h(y)=\frac{1}{|x-y|} \mu\left\{z \in(y, x): \frac{f(z)-f(x)}{z-x}>n\right\}
$$

is clearly continuous on $\left[a_{n}, a_{n+1}\right] \cup\left[b_{n+1}, b_{n}\right]$. Consequently there exists an $\varepsilon_{n}>0$ such that

$$
\mu\left\{z \in(y, x): \frac{f(z)-f(x)}{z-x}>n\right\}>|x-y|\left(\varepsilon_{n}+1 / 2\right)
$$

for each $y \in\left[a_{n}, a_{n+1}\right] \cup\left[b_{n+1}, b_{n}\right]$. Therefore we can find $h_{n}>0$ such that

$$
\mu\left\{z \in(y, x) \backslash\left(-h_{n}, h_{n}\right): \frac{f(z)-f(x)}{z-x}>n\right\}>(1 / 2)|x-y|
$$

for each $y \in\left[a_{n}, a_{n+1}\right] \cup\left[b_{n+1}, b_{n}\right]$. Now we put

$$
E=\left(-\infty, a_{1}\right) \cup\left(b_{1}, \infty\right) \cup \bigcup_{n=1}^{\infty}\left(\left\{z: \frac{f(z)-f(x)}{z-x}>n\right\} \backslash\left(-h_{n}, h_{n}\right)\right) .
$$

It is easy to prove that $E$ has the properties from Definition 2 (with $a=x$ and $A=\infty)$. 
Lemma 3. Let $0 \leq p<a<b \leq 1, K>0$ and $f \in C$. Suppose that, for all $y \in[a, b]$,

$$
\mu\left\{z \in(p, y): \frac{f(z)-f(p)}{z-p}>K\right\}>\frac{1}{2}(y-p) .
$$

Then there exists $0<\eta<a-p$ such that the conditions $\left\|f-f^{*}\right\|<\eta,\left|p^{*}-p\right|<\eta$ and $y \in[a, b]$ imply

$$
\mu\left\{z \in\left(p^{*}, y\right): \frac{f^{*}(z)-f^{*}\left(p^{*}\right)}{z-p^{*}}>K\right\}>\frac{1}{2}\left(y-p^{*}\right) .
$$

Proof. Suppose that no such $\eta$ exists.

Then there exist sequences $f_{n}^{*} \in C, p_{n}^{*} \in[0,1], y_{n} \in[a, b]$ such that $f_{n}^{*} \rightarrow f$ in $C, p_{n}^{*} \rightarrow p$ and

$$
\mu\left(\left(p_{n}^{*}, y_{n}\right) \cap A_{n}\right) \leq \frac{1}{2}\left(y_{n}-p_{n}^{*}\right)
$$

where

$$
A_{n}=\left\{z \in[0,1]: \frac{f_{n}^{*}(z)-f_{n}^{*}\left(p_{n}^{*}\right)}{z-p_{n}^{*}}>K\right\} .
$$

We can and will suppose that $y_{n} \rightarrow y$, where $y \in[a, b]$. Putting

$$
A=\left\{z \in[0,1]: \frac{f(z)-f(p)}{z-p}>K\right\}
$$

we know that

$$
\mu(A \cap(p, y))>\frac{1}{2}(y-p) .
$$

But for each fixed $z \in(p, y)$ obviously

$$
\frac{f_{n}^{*}(z)-f_{n}^{*}\left(p_{n}^{*}\right)}{z-p_{n}^{*}} \rightarrow \frac{f(z)-f(p)}{z-p}
$$

and therefore

$$
A \cap(p, y) \subset \liminf _{n \rightarrow \infty}\left(A_{n} \cap(p, y)\right) .
$$

Using (7) and (5) we obtain

$$
\begin{aligned}
\mu(A \cap(p, y)) & \leq \liminf _{n \rightarrow \infty} \mu\left(A_{n} \cap(p, y)\right) \\
& \leq \limsup _{n \rightarrow \infty}\left(\mu\left(A_{n} \cap\left(p_{n}^{*}, y_{n}\right)\right)+\left|p_{n}^{*}-p\right|+\left|y_{n}^{*}-y\right|\right) \\
& \leq \limsup _{n \rightarrow \infty} \frac{1}{2}\left(y_{n}-p_{n}^{*}\right)=\frac{1}{2}(y-p),
\end{aligned}
$$

which contradicts (6). 


\section{MAIN RESUlT}

Theorem. For a typical $f \in C$ the sets $\left\{x: f_{p r}^{\prime}(x)=\infty\right\}$ and $\left\{x: f_{p r}^{\prime}(x)=\right.$ $-\infty\}$ are c-dense in $(0,1)$.

Proof. Since the mapping $h \rightarrow-h$ is a homeomorphism of $C$ on $C$ and $h_{p r}^{\prime}(x)=$ $-\infty$ iff $(-h)_{p r}^{\prime}(x)=\infty$, it is sufficient to prove that $\left\{x: f_{p r}^{\prime}(x)=\infty\right\}$ is $c$-dense for a typical $f \in C$. Consequently it is sufficient to prove that for each fixed interval $(\alpha, \beta)$ with rational endpoints the set

$$
Q:=\left\{f \in C: \operatorname{card}\left\{x \in(\alpha, \beta): f_{p r}^{\prime}(x)=\infty\right\}=c\right\}
$$

is residual.

By Theorem BM it is sufficient to find a winning strategy for the second player in the Banach-Mazur game for $P=C$ and $Q$. To describe this strategy, put $S_{n}=\{0,1\}^{n}$ and $S=\{0,1\}^{\mathbb{N}}$, where $n \in \mathbb{N}$ and $\mathbb{N}=\{1,2, \ldots\}$. By our strategy the second player will construct in his $n$th move not only a ball $B\left(f_{n}, \varepsilon_{n}\right) \subset C$ but also numbers $\xi_{n}>0, q_{n}>0$ and $2^{n}$ points $\left\{x_{n}^{s}: s \in S_{n}\right\}$ such that the following conditions (8)-(13) hold (where we have put $a_{n}^{s}=x_{n}^{s}-50 \xi_{n}, b_{n}^{s}=x_{n}^{s}+50 \xi_{n}$ ):

(8) $\quad\left\{\left[a_{n}^{s}, b_{n}^{s}\right]: s \in S_{n}\right\}$ are pairwise disjoint subintervals of $(\alpha, \beta)$;

if we fix an arbitrary $s=\left(s_{1}, \ldots, s_{n}\right) \in S_{n}$ and denote $x_{k}=x_{k}^{\left(s_{1}, \ldots, s_{k}\right)}, a_{k}=$ $a_{k}^{\left(s_{1}, \ldots s_{k}\right)}$ and $b_{k}=b_{k}^{\left(s_{1}, \ldots, s_{k}\right)}$ for $1 \leq k \leq n$, then

$$
\left[a_{n}, b_{n}\right] \subset\left(x_{n-1}-4 \xi_{n-1}, x_{n-1}+4 \xi_{n-1}\right) \text { for } n>1 ;
$$

$$
f_{n} \text { is linear on }\left[x_{n}-5 \xi_{n}, x_{n}+5 \xi_{n}\right] \text {, constant on the intervals }
$$

$$
\left[a_{n}, x_{n}-5 \xi_{n}\right],\left[x_{n}+5 \xi_{n}, b_{n}\right] \text { and } \frac{f_{n}\left(b_{n}\right)-f_{n}\left(a_{n}\right)}{b_{n}-a_{n}}=q_{n}>10(n+1) ;
$$

if $n>1$, then for each $x \in\left[x_{n}-4 \xi_{n}, x_{n}+4 \xi_{n}\right]$ and $f \in B\left(f_{n}, \varepsilon_{n}\right)$ we have

(11) $\mu\left\{z \in(y, x): \frac{f(x)-f(z)}{x-z}>n-1\right\}>\frac{1}{2}(x-y)$ for $y \in\left[a_{n-1}, a_{n}\right]$ and

$$
\begin{gathered}
\mu\left\{z \in(x, y): \frac{f(z)-f(x)}{z-x}>n-1\right\}>\frac{1}{2}(y-x) \text { for } y \in\left[b_{n}, b_{n-1}\right] \\
\overline{B\left(f_{n}, \varepsilon_{n}\right)} \subset B\left(g_{n}, \delta_{n}\right) \text { and } \varepsilon_{n}<\frac{1}{2} \xi_{n} .
\end{gathered}
$$

If we prove that the second player can play according to this strategy in all moves, we shall be done. In fact, (9) and (13) imply that $\xi_{n} \rightarrow 0, \varepsilon_{n} \rightarrow 0, \bigcap_{n=1}^{\infty} B\left(f_{n}, \varepsilon_{n}\right)$ consists of one function $f$ and the set $\bigcap_{n=1}^{\infty}\left[a_{n}^{\left(s_{1}, \ldots, s_{n}\right)}, b_{n}^{\left(s_{1}, \ldots, s_{n}\right)}\right]$ consists of one point $x_{s} \in(a, b)$ for each $s=\left(s_{1}, s_{2}, \ldots\right) \in S$. Lemma 2, (9), (11) and (12) 
easily imply that $f_{p r}^{\prime}\left(x_{s}\right)=\infty$. Since (8) implies that $x_{s} \neq x_{s^{*}}$ for $s \neq s^{*}$, we obtain $f \in Q$.

Thus suppose that, for a fixed natural number $m$, the second player has played $m-1$ moves and the first player has played $m$ moves such that the conditions (8)-(13) hold for each $n<m$. We know that $B\left(g_{m}, \delta_{m}\right) \subset B\left(f_{m-1}, \varepsilon_{m-1}\right)$ (if $m>1$ ) and we can clearly suppose without any loss of generality that $g_{m}$ is a piece-wise linear function. Our task is to construct

$$
f_{m}, \varepsilon_{m}, \xi_{m}, q_{m} \text { and }\left\{x_{m}^{s}: s \in S_{m}\right\}
$$

such that the conditions (8)-(13) hold for $n=m$.

If $m=1$, then the conditions (9), (11) and (12) are trivially satisfied and the construction of $f_{1}, \varepsilon_{1}, \xi_{1}, q_{1}$ and $x_{1}^{(0)}, x_{1}^{(1)}$ such that the conditions (8), (10) and (13) are satisfied for $n=1$ is quite easy.

If $m>1$, we shall consider an arbitrary $\left(s_{1}, \ldots, s_{m-1}\right) \in S_{m-1}$ and the function $g_{m}$ on the interval

$$
\left[a_{m-1}, b_{m-1}\right]:=\left[a_{m-1}^{\left(s_{1}, \ldots, s_{m-1}\right)}, b_{m-1}^{\left(s_{1}, \ldots, s_{m-1}\right)}\right]
$$

Since (13) implies $\left\|g_{m}-f_{m-1}\right\|<\varepsilon_{m-1}<\frac{1}{2} \xi_{m-1}<\frac{1}{2}\left(b_{m-1}-a_{m-1}\right)$, we have

$$
\frac{g_{m}\left(b_{m-1}\right)-g_{m}\left(a_{m-1}\right)}{b_{m-1}-a_{m-1}}>\frac{f_{m-1}\left(b_{m-1}\right)-f_{m-1}\left(a_{m-1}\right)}{b_{m-1}-a_{m-1}}-1=q_{m-1}-1 .
$$

Since $g_{m}$ is piece-wise linear, Lemma 1 ( used for $\varphi=g_{m},[a, b]=\left[a_{m-1}, b_{m-1}\right]$, $\left.K=q_{m-1}-1\right)$ implies that we can choose two points $x_{m}^{\left(s_{1}, \ldots, s_{m-1}, 0\right)}, x_{m}^{\left(s_{1}, \ldots, s_{m-1}, 1\right)}$ in $\left(a_{m-1}, b_{m-1}\right)$ such that, denoting any of them by $x_{m}$, the following assertions hold :

$$
g_{m} \text { is linear on a neighbourhood of } x_{m}
$$

for each $y \in\left(x_{m}, b_{m-1}\right]$ we have

$$
\mu\left\{z \in\left(x_{m}, y\right): \frac{g_{m}(z)-g_{m}\left(x_{m}\right)}{z-x_{m}}>q_{m-1}-1\right\}>\frac{1}{2}\left(y-x_{m}\right) \text { and }
$$

$$
\text { for each } y \in\left[a_{m-1}, x_{m}\right) \text { we have }
$$

$$
\mu\left\{z \in\left(y, x_{m}\right): \frac{g_{m}(z)-g_{m}\left(x_{m}\right)}{z-x_{m}}>q_{m-1}-1\right\}>\frac{1}{2}\left(x_{m}-y\right) .
$$

Thus we have constructed for each $s \in S_{m}$ a point $x_{m}^{s}$ (denoted above as $\left.x_{m}\right)$. In the following we shall suppose that $s=\left(s_{1}, \ldots, s_{m}\right)$ is fixed and we shall put for brevity as above

$$
a_{m-1}=a_{m-1}^{\left(s_{1}, \ldots, s_{m-1}\right)}, b_{m-1}=b_{m-1}^{\left(s_{1}, \ldots, s_{m-1}\right)}, x_{m-1}=x_{m-1}^{\left(s_{1}, \ldots, s_{m-1}\right)}, x_{m}=x_{m}^{s} .
$$


Now we shall observe that

$$
x_{m} \in\left(x_{m-1}-4 \xi_{m-1}, x_{m-1}+4 \xi_{m-1}\right) .
$$

In fact, suppose that (17) does not hold. We can suppose without any loss of generality that $x_{m} \geq x_{m-1}$, the case $x_{m} \leq x_{m-1}$ being quite symmetrical. Then

$$
x_{m} \in\left[x_{m-1}+4 \xi_{m-1}, x_{m-1}+5 \xi_{m-1}\right] \quad \text { or } \quad x_{m} \in\left[x_{m-1}+5 \xi_{m-1}, b_{m-1}\right] .
$$

In the first case (10) yields

$$
\begin{aligned}
f_{m-1}\left(x_{m}\right) & \geq f_{m-1}\left(x_{m-1}+4 \xi_{m-1}\right) \\
& =f_{m-1}\left(x_{m-1}\right)+\frac{4}{10}\left(f_{m-1}\left(b_{m-1}\right)-f_{m-1}\left(a_{m-1}\right)\right) \\
& =f_{m-1}\left(x_{m-1}\right)+40 q_{m-1} \xi_{m-1} .
\end{aligned}
$$

For each $z \in\left[x_{m-1}+25 \xi_{m-1}, b_{m-1}\right]$ we have by (10)

$$
f_{m-1}(z)=f_{m-1}\left(b_{m-1}\right)=f_{m-1}\left(x_{m-1}\right)+50 q_{m-1} \xi_{m-1} \text { and } z-x_{m} \geq 20 \xi_{m-1} \text {; }
$$

consequently

$$
\frac{f_{m-1}(z)-f_{m-1}\left(x_{m}\right)}{z-x_{m}} \leq \frac{10 q_{m-1} \xi_{m-1}}{20 \xi_{m-1}}=\frac{q_{m-1}}{2} .
$$

Since $\left\|g_{m}-f_{m-1}\right\|<\frac{1}{2} \xi_{m-1} \quad$ we have

$$
\frac{g_{m}(z)-g_{m}\left(x_{m}\right)}{z-x_{m}} \leq \frac{q_{m-1}}{2}+\frac{\xi_{m-1}}{20 \xi_{m-1}}<\frac{q_{m-1}+1}{2}<q_{m-1}-1
$$

for each $z \in\left[x_{m-1}+25 \xi_{m-1}, b_{m-1}\right]$. This clearly contradicts (15) for $y=b_{m-1}$.

If $x_{m} \in\left[x_{m-1}+5 \xi_{m-1}, b_{m-1}\right]$, we can obviously find

$$
y \in\left[x_{m-1}+5 \xi_{m-1}, x_{m-1}+50 \xi_{m-1}\right] \text { such that }\left|y-x_{m}\right|=10 \xi_{m-1} .
$$

For each $z \in\left(\frac{x_{m}+y}{2}, y\right)$ we have $\left|z-x_{m}\right| \geq 5 \xi_{m-1}$. Thus, since $f_{m-1}(z)=$ $f_{m-1}\left(x_{m}\right)$ and $\left\|g_{m}-f_{m-1}\right\|<\frac{1}{5} \xi_{m-1}$, we have

$$
\frac{g_{m}(z)-g_{m}\left(x_{m}\right)}{z-x_{m}} \leq \frac{\xi_{m-1}}{5 \xi_{m-1}}=\frac{1}{5}<q_{m-1}-1 .
$$

This clearly contradicts (15) (if $y>x_{m}$ ) or (16) (if $y<x_{m}$ ).

Further observe that (14), (15) and (17) yield the existence of $v=v^{s}$ such that

$$
\begin{aligned}
& g_{m} \text { is linear on }\left[x_{m}-v^{s}, x_{m}+v^{s}\right] \subset\left(x_{m-1}-4 \xi_{m-1}, x_{m-1}+4 \xi_{m-1}\right) \\
& \text { with a slope } q=q^{s}>q_{m-1}-1 \text { and the intervals } \\
& \left\{\left[x_{m}^{s}-v^{s}, x_{m}^{s}+v^{s}\right]: s \in S_{m}\right\} \quad \text { are pairwise disjoint. }
\end{aligned}
$$


Using (15), we can apply Lemma 3 to

$$
f=g_{m}, p=x_{m}, a=x_{m}+v, b=b_{m-1}, K=q_{m-1}-1
$$

and obtain $\eta=\eta^{s}>0$ such that:

$$
\begin{aligned}
& \text { whenever }\left\|h-g_{m}\right\|<\eta \text { and }\left|x-x_{m}\right|<\eta, \text { then } \\
& \mu\left\{z \in(x, y): \frac{h(z)-h(x)}{z-x}>q_{m-1}-1\right\}>\frac{1}{2}(y-x) \\
& \text { for all } y \in\left[x_{m}+v, b_{m-1}\right] .
\end{aligned}
$$

Using (16) and the symmetrical version of Lemma 3 , we obtain $\bar{\eta}=\bar{\eta}^{s}>0$ such that:

$$
\begin{gathered}
\text { whenever }\left\|h-g_{m}\right\|<\bar{\eta} \text { and }\left|x-x_{m}\right|<\bar{\eta} \text {, then } \\
\mu\left\{z \in(y, x): \frac{h(z)-h(x)}{z-x}>q_{m-1}-1\right\}>\frac{1}{2}(x-y) \\
\text { for all } y \in\left[a_{m-1}, x_{m}-v\right] .
\end{gathered}
$$

Now choose $q_{m}$ so big and $\xi_{m}>0$ so small (note that $q_{m}$ and $\xi_{m}$ do not depend on $s$ ) that for each $s \in S_{m}$

$$
\begin{gathered}
q_{m}>\max \left(q^{s}, 10(m+1)\right), \\
50 \xi_{m}<\min \left(\frac{\eta^{s}}{2 q_{m}}, \frac{\bar{\eta}^{s}}{2 q_{m}}\right), \\
50 \xi_{m}<\frac{q^{s} v^{s}}{q_{m}} \quad \text { and }
\end{gathered}
$$

On account of (21) we obtain $5 \xi_{m}<\frac{50 \xi_{m} q_{m}}{q^{s}}$ and (23) implies that

$$
I_{m}^{s}:=\left[x_{m}^{s}-\frac{50 \xi_{m} q_{m}}{q^{s}}, x_{m}^{s}+\frac{50 \xi_{m} q_{m}}{q^{s}}\right] \subset\left[x_{m}^{s}-v^{s}, x_{m}^{s}+v^{s}\right] .
$$

Consequently (18) gives that $\left\{I_{m}^{s}: s \in S_{m}\right\} \quad$ are pairwise disjoint intervals.

On each interval $I_{m}^{s}$ we now define the function $f_{m}$ by the following conditions:

$$
f_{m}\left(x_{m}^{s}\right)=g_{m}\left(x_{m}^{s}\right)
$$

$f_{m}$ is linear on $\left[x_{m}^{s}-5 \xi_{m}, x_{m}^{s}+5 \xi_{m}\right]$ with the slope $10 q_{m}$,

$$
f_{m}(x)=g_{m}\left(x_{m}^{s}\right)+50 q_{m} \xi_{m} \text { for } x \in\left[x_{m}^{s}+5 \xi_{m}, x_{m}^{s}+\frac{50 \xi_{m} q_{m}}{q^{s}}\right] \text { and }
$$




$$
f_{m}(x)=g_{m}\left(x_{m}^{s}\right)-50 q_{m} \xi_{m} \text { for } x \in\left[x_{m}^{s}-\frac{50 \xi_{m} q_{m}}{q^{s}}, x_{m}^{s}-5 \xi_{m}\right] .
$$

For $x \in[0,1]$ which belongs to no interval $I_{m}^{s}$, we put $f_{m}(x)=g_{m}(x)$.

Using (18) we easily obtain that $f_{m} \in C$. On account of (18) and the definition of $f_{m}$ we easily see that $\left\|f_{m}-g_{m}\right\| \leq 50 q_{m} \xi_{m}$. Consequently (24) implies $f_{m} \in$ $B\left(g_{m}, \delta_{m}\right)$. Thus we can choose an $\varepsilon_{m}>0$ such that

$$
\begin{gathered}
\overline{B\left(f_{m}, \varepsilon_{m}\right)} \subset B\left(g_{m}, \delta_{m}\right), \varepsilon_{m}<\frac{\xi_{m}}{2} \\
\text { and } \quad \varepsilon_{m}<\min \left(\frac{\eta^{s}}{2}, \frac{\bar{\eta}^{s}}{2}\right) \quad \text { for each } \quad s \in S_{m} .
\end{gathered}
$$

Now we have to show that the conditions (8) - (13) hold for $n=m$.

By (21) we have $\left[a_{m}^{s}, b_{m}^{s}\right] \subset I_{m}^{s}$, which implies (8).

In the following we shall suppose that an arbitrary $s \in S_{m}$ is fixed and we shall omit the index $s$.

The condition (9) easily follows from (18), since

$$
\left[a_{m}, b_{m}\right] \subset I_{m} \subset\left[x_{m}-v, x_{m}+v\right] .
$$

Since $\left[a_{m}, b_{m}\right] \subset I_{m}$, the condition (10) follows immediately from the definition of $f_{m}$.

The condition (13) is contained in (25).

Thus it is sufficient to prove (11) and (12). We shall prove (12) only, the proof of (11) being symmetrical. To prove (12), consider arbitrary

$$
x \in\left[x_{m}-4 \xi_{m}, x_{m}+4 \xi_{m}\right], f \in B\left(f_{m}, \varepsilon_{m}\right) \text { and } y \in\left[b_{m}, b_{m-1}\right] .
$$

We shall distinguish two possibilities :

$$
y \in\left[x_{m}+v, b_{m-1}\right] \text { and } y \in\left[b_{m}, x_{m}+v\right) .
$$

In the first case (22) gives

$$
\left\|f_{m}-g_{m}\right\| \leq 50 q_{m} \xi_{m}<\frac{\eta}{2}
$$

Consequently (25) implies $\left\|f-g_{m}\right\|<\eta$. By (22) also $\left|x-x_{m}\right|<\eta$ and consequently (19) and (10) yield

$$
\mu\left\{z \in(x, y): \frac{f(z)-f(x)}{z-x}>10 m-1\right\}>\frac{1}{2}(y-x) .
$$

In the second case we shall estimate $\frac{f(z)-f(x)}{z-x}$ separately for the cases

$$
z \in\left[x_{m}+5 \xi_{m}, x_{m}+\frac{50 \xi_{m} q_{m}}{q}\right] \quad \text { and }
$$

$$
z \in\left(x_{m}+\frac{50 \xi_{m} q_{m}}{q}, x_{m}+v\right)
$$


In the case $(27)$ we have

$$
f_{m}(x) \leq f_{m}\left(x_{m}+4 \xi_{m}\right)=f_{m}\left(x_{m}\right)+40 q_{m} \xi_{m}, \quad f_{m}(z)=f_{m}\left(x_{m}\right)+50 q_{m} \xi_{m}
$$

and consequently $f_{m}(z)-f_{m}(x)>10 q_{m} \xi_{m}$. Since

$$
z-x<\frac{50 \xi_{m} q_{m}}{q}+4 \xi_{m} \quad \text { and } \quad\left\|f_{m}-f\right\|<\frac{1}{2} \xi_{m}
$$

we obtain

$$
\frac{f(z)-f(x)}{z-x}>\frac{10 q_{m} \xi_{m}-\xi_{m}}{\frac{50 \xi_{m} q_{m}}{q}+4 \xi_{m}}>\frac{9 q_{m}}{54 \frac{q_{m}}{q}}=\frac{q}{6}>\frac{1}{6}\left(q_{m-1}-1\right)>\frac{10 m-1}{6}>m .
$$

In the case (28) observe that we have proved

$$
\frac{f_{m}\left(x_{m}+\frac{50 \xi_{m} q_{m}}{q}\right)-f_{m}(x)}{\left(x_{m}+\frac{50 \xi_{m} q_{m}}{q}\right)-x}>\frac{q}{6}
$$

$$
\frac{f_{m}(z)-f_{m}\left(x_{m}+\frac{50 \xi_{m} q_{m}}{q}\right)}{z-\left(x_{m}+\frac{50 \xi_{m} q_{m}}{q}\right)}=q \quad \text { and consequently }
$$

$\frac{f_{m}(z)-f_{m}(x)}{z-x}>\frac{q}{6}$. Since $\quad\left\|f_{m}-f\right\|<\frac{1}{2} \xi_{m} \quad$ and $\quad z-x>45 \xi_{m}$, we have

$$
\frac{f(z)-f(x)}{z-x}>\frac{q}{6}-1>\frac{10 m-1}{6}-1>m .
$$

Consequently we have

$$
\mu\left\{z \in(x, y): \frac{f(z)-f(x)}{z-x}>m\right\} \geq \mu\left[x_{m}+5 \xi_{m}, y\right]>\frac{1}{2}(y-x) .
$$

From (29) and (26) we obtain (12) which completes the proof.

\section{REFERENCES}

1. S. Banach, Über die Differenzierbarkeit stetiger Funktionen, Studia Math. 3 (1931), 174-179.

2. A. M. Bruckner, The Differentiability properties of typical functions in $C[a, b]$, Amer. Math. Monthly 80 (1973), 679-683. MR 47:6956

3. __ Some observations about Denjoy's preponderant derivative, Bull. Math. Soc. Sci. Math. R. S. Roumanie 21(69) (1977), 1-10. MR 57:9922

4. A. Denjoy, Mémoire sur la totalisation des nombres dérivées nonsommables, Ann. École Norm Sup. 33, 34 (1916, 1917), 127-236, 181-238.

5. V. Jarník, Sur la dérivabilité des fonctions continues, Publications de la Fac. des Sc. de L'Univ. Charles 129 (1934), 9 pp.

6. J. Malý, D. Preiss, and L. Zajíček, An unusual monotonicity theorem with applications, Proc. Amer. Math. Soc. 102 (1988), 925-932. MR 89e:26024

7. S. Mazurkiewicz, Sur les fonctions non dérivables, Studia Math. 3 (1931), 92-94.

8. J. L. Leonard, Some conditions implying the monotonicity of a real function, Rev. Roumaine Math. Pures Appl. 17 (1972), 757-780. MR 46:3709

9. J. Oxtoby, The Banach-Mazur game and Banach category theorem, in: Contributions to the Theory of Games III, Ann. of Math. Stud. 39 (1957), 159-163. MR 20:264

10. Measure and category, Springer-Verlag, New York, 1980. MR 81j:28003

11. L. Zajiček, The differentiability structure of typical functions in $C[0,1]$, Real Analysis Exchange 13 (1987-88), 119, 103-106, 93.

Department of Mathematical Analysis, Charles University, Sokolovská 83, 18600 Praha 8, Czech Republic

E-mail address: Zajicek@karlin.mff.cuni.cz 